\title{
Operation Transform Formulae for the Generalized Canonical Hartley Transform
}

\author{
A. S. Gudadhe ${ }^{\#}$ And A.V. Joshi * \\ \# Govt. Vidarbha Institute of Science and Humanities, Amravati. (M. S.) \\ * Shankarlal Khandelwal Arts, Science,\& Commerece College, Akola - 444002 (M. S.)
}

\begin{abstract}
The canonical Hartley transform (CHT) is one of the important transform in the class of linear canonical transform (LCT). It has been used in several areas, including optical analysis and signal processing. For practical purpose canonical Hartley transform is more useful. Hence in this paper we have proved its Inversion theorem along with uniqueness. Some important results about Differentiation, Linearity, Shifting, Scaling property for canonical Hartley transform are also explored.
\end{abstract}

Keywords: Linear canonical transform, Hartley Transform, Fractional Fourier Transform.

\section{Introduction:}

The idea of the fractional powers of Fourier operator appeared in mathematical literature as early in 1930. It has been rediscovered in quantum mechanics by Namias [7]. He had given a systematic method for the development of fractional integral transforms by means of Eigen values. Last decades, since Namias in 1980 develop the eigenvalue methods for Fractional Fourier transform, number of other integral transform have been extended in its fractional domain. For examples Almeida [1] had studied fractional Fourier transform, Fractional Hilbert transform has been developed by Sontakke [10] studied number of property of fractional Hartley transform, Joshi, Gudadhe [3,4] studied number of property of generalized canonical sine transform, also Operation Transform Formulae for the Generalized Half Canonical Sine Transform etc. Bhosale and Choudhary [2] had studied it as a tempered distribution; number of applications of fractional Fourier transforms in signal processing, image processing filtering optics, etc is studied. These fractional transforms found number of applications in signal processing, image processing, quantum mechanics etc.

Further generalization of fractional Fourier transform known as linear canonical transform was introduced by Moshinsky [6] in 1971. Pei, Ding [8,9] had studied its eigen value aspect. Linear canonical transform is a three parameter linear integral transform which has several special cases as fractional Fourier transform, Fresnel transform, Chirp transform etc. Linear canonical transform is defined as,

$$
\begin{gathered}
{[\operatorname{LCTf}(t)](s)=\sqrt{\frac{1}{2 \pi i b}} \int_{-\infty}^{\infty} e^{\frac{i}{2}\left(\frac{d}{b}\right) s^{2}} \cdot e^{\frac{i}{2}(a / b) t^{2}} \cdot e^{-i(s / b) t} f(t) d t, \quad \text { for } \mathrm{b} \neq 0} \\
=\sqrt{d} e^{\frac{i}{2}(c d) s^{2}} \cdot \mathrm{f}(\mathrm{d} \cdot \mathrm{s}), \text { for } \mathrm{b}=0, \text { with ad }-\mathrm{bc}=1,
\end{gathered}
$$

where $\mathrm{a}, \mathrm{b}, \mathrm{c}$, and $\mathrm{d}$ are real parameters independent on $\mathrm{s}$ and $\mathrm{t}$.

Now a day in optical image processing, image encryption technique has great importance and fractional Fourier transform, Fresnel transform, Wavelet transform are some of the transformation used for this. Fractional Hartley transform is also suggested for optical image processing and encryption by Jimenez in [5]. Canonical Hartley transform which is generalization of Fractional Hartley transform with more parameter can be the perfect substitute for that. Hence we study it in the generalized sense and developed its operation relations which are generally used while solving differential equations

In this paper first we have defined generalized Canonical Hartley transform. We have proved some important results about Inversion theorem, Differentiation, Linearity, Shifting, Scaling property for canonical Hartley transform.

\subsection{Testing Function Space $\mathcal{E}$ :}

\section{Generalized Canonical Hartley transform}

An infinitely differentiable complex valued function $\phi$ on $R^{n}$ belongs to $\mathcal{E}\left(R^{n}\right)$. If for each compact set, $I \subset S_{\alpha}$ where $S_{\alpha}=\left\{t: t \in R^{n},|t| \leq \alpha, \alpha>0\right\}$ and for $k \in R^{n}$,

$$
\gamma_{\mathcal{E}, k} \varphi(t)=\sup _{t \in I}\left|D^{k} \varphi(t)\right|<\infty
$$


Note that space $\mathcal{E}$ is complete and a Frechet space, let $\mathcal{E}$ ' denotes the dual space of $\mathcal{E}$.

\subsection{The Canonical Hartley transform on $\mathcal{E}$ :}

The Canonical Hartley transform $f \in \mathcal{E}^{\prime}\left(R^{n}\right)$ can be defined by, $\{C H T f(t)\}(s)=\langle f(t), K(t, s)\rangle$ where,

$$
K(t, s)=\sqrt{\frac{1}{2 \pi i b}} e^{\frac{i}{2} \frac{d}{b} s^{2}} e^{\frac{i}{2} \frac{a}{b} t^{2}}\left(\cos \left(\frac{s}{b} t\right)+\sin \left(\frac{s}{b} t\right)\right)
$$

Hence the canonical Hartley transform $f \in \mathcal{E}^{\prime}\left(R^{n}\right)$ can be defined by,

$[C H T f(t)](s)=\sqrt{\frac{1}{2 \pi i b}} e^{\frac{i}{2} \frac{d}{b} s^{2}} \int_{-\infty}^{\infty} e^{\frac{i}{2} \frac{a}{b} t^{2}}\left(\cos \left(\frac{s}{b} t\right)+\sin \left(\frac{s}{b} t\right)\right) f(t) d t$.

\subsection{Inversion theorem for canonical Hartley transform :}

If $\{C H T f(t)\}(s)$ canonical Hartley transform of $f(t)$ is given by,

$$
\begin{gathered}
\{\text { CHT } f(t)\}(s)=\sqrt{\frac{1}{2 \pi i b}} e^{\frac{i}{2}\left(\frac{d}{b}\right) s^{2}} \int_{-\infty}^{\infty} e^{\frac{i}{2}\left(\frac{a}{b}\right) t^{2}} \operatorname{cas}\left(\frac{s}{b} t\right) f(t) d t \\
\text { then, } f(t)=\sqrt{\frac{2 \pi i}{b}} e^{-\frac{i}{2}\left(\frac{a}{b}\right) t^{2}} \int_{-\infty}^{\infty} e^{-\frac{i}{2}\left(\frac{d}{b}\right) s^{2}} \operatorname{cas}\left(\frac{s}{b} t\right) F(s) d s
\end{gathered}
$$

Proof: The canonical Hartley transform of $f(t)$ is given by

$$
\begin{gathered}
\{C H T f(t)\}(s)=\sqrt{\frac{1}{2 \pi i b}} e^{\frac{i}{2}\left(\frac{d}{b}\right) s^{2}} \int_{-\infty}^{\infty} e^{\frac{i}{2}\left(\frac{a}{b}\right) t^{2}} \operatorname{cas}\left(\frac{s}{b} t\right) f(t) d t \\
F(s)=\frac{1}{\sqrt{2 \pi i b}} e^{\frac{i}{2}\left(\frac{d}{b}\right) s^{2}} \int_{-\infty}^{\infty} e^{\frac{i}{2}\left(\frac{a}{b}\right) t^{2}} \operatorname{cas}\left(\frac{s}{b} t\right) f(t) d t \\
\text { where, }\{C H T f(t)\}(s)=F(s)
\end{gathered}
$$

$\therefore F(s) \sqrt{2 \pi i b} e^{-\frac{i}{2}\left(\frac{d}{b}\right) s^{2}}=\int_{-\infty}^{\infty} e^{\frac{i}{2}\left(\frac{a}{b}\right) t^{2}} \operatorname{cas}\left(\frac{s}{b} t\right) f(t) d t$

$\therefore C_{1}(s)=\int_{-\infty}^{\infty} g(t) \operatorname{cas}\left(\frac{s}{b} t\right) d t$

where, $\quad C_{1}(s)=F(s) \sqrt{2 \pi i b} \cdot e^{-\frac{1}{2}\left(\frac{d}{b}\right) s^{2}}$

$$
\text { and } g(t)=e^{\frac{i}{2}\left(\frac{a}{b}\right) t^{2}} f(t)
$$

$C_{1}(s)=\int_{-\infty}^{\infty} g(t) \operatorname{cas}\left(\frac{s}{b} t\right) d t$

$\because \frac{s}{b}=\eta \quad \Rightarrow b d \eta=d s$

$\therefore C_{1}(s)=\int_{-\infty}^{\infty} g(t) \operatorname{cas}(\eta t) d \eta$

$\therefore$ By using inverse formula.

$\therefore g(t)=\int_{-\infty}^{\infty} C_{1}(s) \operatorname{cas}(\eta t) d \eta$ 


$$
\begin{gathered}
\therefore e^{\frac{i}{2}\left(\frac{a}{b}\right) t^{2}} f(t)=\int_{-\infty}^{\infty} F(s) \sqrt{2 \pi i b} e^{-\frac{i}{2}\left(\frac{d}{b}\right) s^{2}} \operatorname{cas}(\eta t) d \eta \\
f(t)=e^{-\frac{i}{2}\left(\frac{a}{b}\right) t^{2}} \int_{-\infty}^{\infty} e^{-\frac{i}{2}\left(\frac{d}{b}\right) s^{2}} \sqrt{2 \pi i b} F(s) \operatorname{cas}(\eta t) \frac{d s}{b} \\
f(t)=\sqrt{\frac{2 \pi i}{b}} e^{-\frac{i}{2}\left(\frac{a}{b}\right) t^{2}} \int_{-\infty}^{\infty} e^{-\frac{i}{2}\left(\frac{d}{b}\right) s^{2}} \operatorname{cas}\left(\frac{s}{b} t\right) F(s) d s
\end{gathered}
$$

\subsection{Linearity properties for canonical Hartley transform:}

If $\{C H T f(t)\}(s),\{C H T g(t)\}(s)$ denotes generalized canonical Hartley transforms of $f(t), g(t)$ and $\mathrm{P}_{1}, \mathrm{P}_{2}$ are constants then,

$\left\{C H T\left[P_{1} f(t)+P_{2} g(t)\right]\right\}(s)=P_{1}\{C H T f(t)\}(s)+P_{2}\{C H T g(t)\}(s)$

Proof: The proof is simple and hence omitted.

\subsection{Differentiation property of Canonical Hartley Transform:}

If $\{C H T f(t)\}(s)$ denotes generalized canonical Hartley transform of $f(t)$ then

$\left\{C H T\left(f^{\prime}(t)\right)\right\}(s)=\left(\frac{s}{b}\right)\{C H T f(t)\}(s)-i\left(\frac{a}{b}\right)\{C H T t f(t)\}(s)$

Proof: We have, $\left\{C H T f^{\prime}(t)\right\}(s)=\sqrt{\frac{1}{2 \pi i b}} e^{\frac{i}{2}\left(\frac{d}{b}\right) s^{2}} \int_{-\infty}^{\infty} e^{\frac{i}{2}\left(\frac{a}{b}\right) t^{2}} \operatorname{cas}\left(\frac{s}{b} t\right) f^{\prime}(t) d t$

By integration by parts, we get,

$$
\left\{C H T f^{\prime}(t)\right\}(s)=\sqrt{\frac{1}{2 \pi i b}} \cdot e^{\frac{i}{2}\left(\frac{d}{b}\right) s^{2}}\left\{\left[e^{\frac{i}{2}\left(\frac{a}{b}\right) t^{2}} \operatorname{c} a \mathrm{~s}\left(\frac{s}{b} t\right) f(t)\right]_{-\infty}^{\infty}-\left[\int_{-\infty}^{\infty} \frac{\partial}{\partial t}\left(e^{\frac{i}{2}\left(\frac{a}{b}\right) t^{2}}\left(\cos \left(\frac{s}{b} t\right)+\sin \left(\frac{s}{b} t\right)\right)\right) \cdot \int_{-\infty}^{\infty} f^{\prime}(t) d t\right]\right\}
$$

$$
\begin{aligned}
& =\sqrt{\frac{1}{2 \pi i b}} \cdot e^{\frac{i}{2}\left(\frac{d}{b}\right)^{2}}\left\{\left[\left(e^{\frac{i}{2}\left(\frac{a}{b}\right) t^{2}}\left(\cos \left(\frac{s}{b} t\right)+\sin \left(\frac{s}{b} t\right)\right) f(t)\right]_{-\infty}^{\infty}-\int_{-\infty}^{\infty}\left[e^{\frac{i}{2}\left(\frac{a}{b}\right) t^{2}}\left(-\sin \left(\frac{s}{b} t\right)+\cos \left(\frac{s}{b} t\right)\right)\left(\frac{s}{b}\right)+\left(\cos \left(\frac{s}{b} t\right)+\sin \left(\frac{s}{b} t\right)\right) \frac{\partial}{\partial t}\left(e^{\frac{i}{2}\left(\frac{a}{b}\right)^{2}}\right)\right] f(t) d t\right\}\right. \\
& =\sqrt{\frac{1}{2 \pi i b}} \cdot e^{\frac{i}{2}\left(\frac{d}{b}\right) s^{2}}\left\{0+\left(\frac{s}{b}\right) \int_{-\infty}^{\infty} e^{\frac{i}{2}\left(\frac{a}{b}\right) t^{2}}\left(-\cos \left(\frac{s}{b} t\right)+\sin \left(\frac{s}{b} t\right)\right) f(t) d t-i\left(\frac{a}{b}\right) \int_{-\infty}^{\infty} e^{\frac{i}{2}\left(\frac{a}{b}\right)^{2}}\left(\cos \left(\frac{s}{b} t\right)+\sin \left(\frac{s}{b} t\right)\right) t f(t) d t\right\} \\
& =\sqrt{\frac{1}{2 \pi i b}} \cdot e^{\frac{i}{\left(2\left(\frac{d}{b}\right) s^{2}\right.}}\left\{\left(\frac{s}{b}\right) \int_{-\infty}^{\infty} e^{\frac{i}{2}\left(\frac{a}{b}\right)^{2}}\left(\cos \left(-\frac{s}{b} t\right)+\sin \left(\frac{s}{b} t\right)\right) f(t) d t-i\left(\frac{a}{b}\right) \int_{-\infty}^{\infty} e^{\frac{i}{2}\left(\frac{a}{b}\right) t^{2}}\left(\cos \left(\frac{s}{b} t\right)+\sin \left(\frac{s}{b} t\right)\right) t f(t) d t\right\} \\
& =\left(\frac{s}{b}\right) \sqrt{\frac{1}{2 \pi i b}} \cdot e^{\frac{i}{2}\left(\frac{d}{b}\right) s^{2}} \int_{-\infty}^{\infty} e^{\frac{i}{2}\left(\frac{a}{b}\right)^{2}}\left(\cos \left(\frac{s}{b} t\right)+\sin \left(\frac{s}{b} t\right)\right) f(t) d t-i\left(\frac{a}{b}\right) \sqrt{\frac{1}{2 \pi i b}} \cdot e^{\frac{i}{2}\left(\frac{d}{b}\right) s^{2}} \int_{-\infty}^{\infty} e^{\frac{i}{2}\left(\frac{a}{b}\right)^{2}}\left(\cos \left(\frac{s}{b} t\right)+\sin \left(\frac{s}{b} t\right)\right) t f(t) d t \\
& \left\{C H T\left(f^{\prime}(t)\right)\right\}(s)=\left(\frac{s}{b}\right)\{C H T f(t)\}(s)-i\left(\frac{a}{b}\right)\{C H T t f(t)\}(s)
\end{aligned}
$$

\subsection{Derivative property of canonical Hartley transform:}

If $\{C H T f(t)\}(s)$ denotes generalized canonical Hartley transform of $f(t)$ then,

Proof: We have,

$$
\frac{d}{d s}\{C H T f(t)\}(s)=i\left(\frac{s d}{b}\right)\{C H T f(t)\}-\left(\frac{1}{b}\right)\{C H T t f(t)\}
$$




$$
\begin{gathered}
\frac{d}{d s}\{C H T f(t)\}(s)=\frac{d}{d s}\left\{\sqrt{\frac{1}{2 \pi i b}} \cdot e^{\frac{i}{2}\left(\frac{d}{b}\right) s^{2}} \int_{-\infty}^{\infty} e^{\frac{i}{2}\left(\frac{a}{b}\right) t^{2}} \operatorname{cas}\left(\frac{s}{b} t\right) f(t) d t\right\} \\
\frac{d}{d s}\{C H T f(t)\}(s)=\sqrt{\frac{1}{2 \pi i b}} \int_{-\infty}^{\infty} e^{\frac{i}{2}\left(\frac{a}{b}\right) t^{2}} \frac{\partial}{\partial s}\left(e^{\frac{i}{2}\left(\frac{d}{b}\right) s^{2}}\left(\cos \left(\frac{s}{b} t\right)+\sin \left(\frac{s}{b} t\right)\right)\right) f(t) d t \\
=\sqrt{\frac{1}{2 \pi i b}} \int_{-\infty}^{\infty} e^{\frac{i}{2}\left(\frac{a}{b}\right) t^{2}}\left(\left(\frac{t}{b}\right) e^{\frac{i}{2}\left(\frac{d}{b}\right)^{s^{2}}}\left(\cos \left(\frac{s}{b} t\right)-\sin \left(\frac{s}{b} t\right)\right)+i\left(\frac{d}{b}\right) s e^{\frac{i}{2}\left(\frac{d}{b}\right) s^{2}}\left(\cos \left(\frac{s}{b} t\right)+\sin \left(\frac{s}{b} t\right)\right)\right) f(t) d t \\
=\sqrt{\frac{1}{2 \pi i b}} \int_{-\infty}^{\infty} e^{\frac{i}{2}\left(\frac{a}{b}\right) t^{2}}\left(-\left(\frac{t}{b}\right) e^{\frac{i}{2}\left(\frac{d}{b}\right) s^{2}}\left(-\cos \left(\frac{s}{b} t\right)+\sin \left(\frac{s}{b} t\right)\right)+i\left(\frac{d}{b}\right) s e^{\frac{i}{2}\left(\frac{d}{b}\right) s^{2}}\left(\cos \left(\frac{s}{b} t\right)+\sin \left(\frac{s}{b} t\right)\right) f(t) d t\right. \\
=\sqrt{\frac{1}{2 \pi i b}} \int_{-\infty}^{\infty} e^{\frac{i}{2}\left(\frac{a}{b}\right) t^{2}}\left(-\left(\frac{t}{b}\right) e^{\frac{i}{2}\left(\frac{d}{b}\right) s^{2}}\left(\cos \left(-\frac{s}{b} t\right)+\sin \left(\frac{s}{b} t\right)\right)+i\left(\frac{d}{b}\right) s e^{\frac{i}{2}\left(\frac{d}{b}\right) s^{2}}\left(\cos \left(\frac{s}{b} t\right)+\sin \left(\frac{s}{b} t\right)\right) f(t) d t\right. \\
=-\sqrt{\frac{1}{2 \pi i b}} \int_{-\infty}^{\infty} e^{\frac{i}{2}\left(\frac{a}{b}\right) t^{2}}\left(\frac{t}{b}\right) e^{\frac{i}{2}\left(\frac{d}{b}\right)^{s^{2}}}\left(\cos \left(\frac{s}{b} t\right)+\sin \left(\frac{s}{b} t\right)\right) f(t) d t+i\left(\frac{d}{b}\right) s \sqrt{\frac{1}{2 \pi i b}} \int_{-\infty}^{\infty} e^{\frac{i}{2}\left(\frac{a}{b}\right) t^{2}} e^{\frac{i}{2}\left(\frac{d}{b}\right) s^{2}}\left(\cos \left(\frac{s}{b} t\right)+\sin \left(\frac{s}{b} t\right)\right) f(t) d t \\
\quad \frac{d}{d s}\{C H T f(t)\}(s)=i\left(\frac{s d}{b}\right)\{C H T f(t)\}-\left(\frac{1}{b}\right)\{C H T t f(t)\}
\end{gathered}
$$

\subsection{Shifting property of canonical Hartley transform:}

If $\{C H T f(t)\}(s)$ denotes generalized canonical Hartley transform of $f(t)$ and ' $\tau$, is any real number. Then,

$$
\{C H T[f(t+\tau)]\}(s)=e^{\frac{i}{2}\left(\frac{a}{b}\right) \tau^{2}}\left[\cos \left(\frac{s}{b} \tau\right)\left\{C H T f(t) \cdot e^{-i t \tau\left(\frac{a}{b}\right)}\right\}(s)+\sin \left(\frac{s}{b} \tau\right)\left\{C H T f(t) \cdot e^{-i t \tau\left(\frac{a}{b}\right)}\right\}(s)\right]
$$

Proof: We have, $\{C H T f(t+\tau)\}(s)=\sqrt{\frac{1}{2 \pi i b}} \cdot e^{\frac{i}{2}\left(\frac{d}{b}\right) s^{2}} \int_{-\infty}^{\infty}\left(\cos \left(\frac{s}{b} t\right)+\sin \left(\frac{s}{b} t\right)\right) e^{\frac{i}{2}\left(\frac{a}{b}\right) t^{2}} \cdot f(t+\tau) d t$

$$
\{C H T f(t+\tau)\}(s)=C_{1}(s) \int_{-\infty}^{\infty}\left(\cos \left(\frac{s}{b} t\right)+\sin \left(\frac{s}{b} t\right)\right) e^{\frac{i}{2}\left(\frac{a}{b}\right) t^{2}} \cdot f(t+\tau) d t
$$

where, $\quad C_{1}(s)=\sqrt{\frac{1}{2 \pi i b}} \cdot e^{\frac{i}{2}\left(\frac{d}{b}\right) s^{2}}$ putting $t=(T-\tau) \quad \Rightarrow d t=d T$

$$
\begin{gathered}
\{\text { CHT } f(t+\tau)\}(s)=C_{1}(s) \int_{-\infty}^{\infty}\left(\cos \left(\frac{s}{b}(T-\tau)\right)+\sin \left(\frac{s}{b}(T-\tau)\right)\right) e^{\frac{i}{2}\left(\frac{a}{b}\right)\left(T^{2}-2 T \tau+\tau^{2}\right)} \cdot f(T) d T \\
\{C H T f(t+\tau)\}(s)=C_{1}(s) \int_{-\infty}^{\infty}\left(\cos \left(\frac{s}{b}(T-\tau)\right)+\sin \left(\frac{s}{b}(T-\tau)\right)\right) e^{\frac{i}{2}\left(\frac{a}{b}\right) T^{2}} \cdot e^{-i T \tau\left(\frac{a}{b}\right)} \cdot e^{\frac{i}{2}\left(\frac{a}{b}\right) \tau^{2}} \cdot f(T) d T \\
\{C H T f(t+\tau)\}(s)=C_{1}(s) \cdot e^{\frac{i}{2}\left(\frac{a}{b}\right) \tau^{2}} \int_{-\infty}^{\infty} \cos \left(\frac{s}{b}(T-\tau)\right) \cdot e^{\frac{i}{2}\left(\frac{a}{b}\right) T^{2}} \cdot e^{-i T \tau\left(\frac{a}{b}\right)} f(T) d T \\
=\sqrt{\frac{1}{2 \pi i b}} \cdot e^{\frac{i}{2}\left(\frac{d}{b}\right) s^{2}} \cdot e^{\frac{i}{2}\left(\frac{a}{b}\right)^{2} \cdot \int_{-\infty}^{\infty} e^{2} \frac{i}{2}\left(\frac{a}{b}\right) T^{2} \cdot} \cdot e^{-i T \tau\left(\frac{a}{b}\right)} . \\
F(T) \cdot\left[\cos \left(\frac{s}{b} T\right) \cdot \cos \left(\frac{s}{b} \tau\right)+\sin \left(\frac{s}{b} T\right) \cdot \sin \left(\frac{s}{b} \tau\right)+\sin \left(\frac{s}{b} T\right) \cos \left(\frac{s}{b} \tau\right)-\cos \left(\frac{s}{b} T\right) \sin \left(\frac{s}{b} \tau\right)\right] d T
\end{gathered}
$$




$$
\begin{gathered}
=e^{\frac{i}{2}\left(\frac{a}{b}\right) \tau^{2}}\left\{\cos \left(\frac{s}{b} \tau\right) C_{1}(s) \int_{-\infty}^{\infty} e^{\frac{i}{2}\left(\frac{a}{b}\right) T^{2}} \cdot e^{-i T \tau\left(\frac{a}{b}\right)} \cdot f(T) \cdot\right. \\
\left.\left(\cos \left(\frac{s}{b} T\right)+\sin \left(\frac{s}{b} T\right)\right) d T+\sin \left(\frac{s}{b} \tau\right) C_{1}(s) \int_{-\infty}^{\infty} e^{\frac{i}{2}\left(\frac{a}{b}\right) T^{2}} \cdot e^{-i T \tau\left(\frac{a}{b}\right)} \cdot f(T)\left(\cos \left(-\frac{s}{b} T\right)+\sin \left(\frac{s}{b} T\right)\right) d T\right\} \\
=e^{\frac{i}{2}\left(\frac{a}{b}\right) \tau^{2}}\left\{\cos \left(\frac{s}{b} \tau\right)\left[\operatorname{CHT} f(t) e^{-i T \tau\left(\frac{a}{b}\right)}\right](s)+\sin \left(\frac{s}{b} \tau\right)\left[\operatorname{CHT} f(t) \cdot e^{-i T \tau\left(\frac{a}{b}\right)}\right](s)\right\} \\
\{C H T[f(t+\tau)]\}(s)=e^{\frac{i}{2}\left(\frac{a}{b}\right) \tau^{2}}\left[\cos \left(\frac{s}{b} \tau\right)\left\{\operatorname{CHT} f(t) \cdot e^{-i t \tau\left(\frac{a}{b}\right)}\right\}(s)+\sin \left(\frac{s}{b} \tau\right)\left\{\operatorname{CHTf} f(t) \cdot e^{-i t \tau\left(\frac{a}{b}\right)}\right\}(s)\right]
\end{gathered}
$$

2.8 Scaling property of canonical Hartley transform:

If $\{C H T f(t)\}(s)$ denotes generalized canonical Hartley transform of $f(t)$ then, $\{C H T[f(k t)]\}(s)=\frac{1}{k} e^{\left(1-\frac{1}{k}\right) \frac{i}{2}\left(\frac{d}{b}\right) s^{2}}\left[C H T\left\{f(t) \cdot e^{\left(\frac{1}{k}-1\right) \frac{i}{2} \frac{a}{b k} t^{2}}\right\}\right]\left(\frac{s}{b k}\right)$

Proof: We have, $\{C H T f(t)\}(s)=\sqrt{\frac{1}{2 \pi i b}} \cdot e^{\frac{i}{2}\left(\frac{d}{b}\right) s^{2}} \int_{-\infty}^{\infty} e^{\frac{i}{2}\left(\frac{a}{b}\right) t^{2}}\left(\cos \left(\frac{s}{b} t\right)+\sin \left(\frac{s}{b} t\right)\right) f(t) d t$

$$
\{C H T f(k t)\}(s)=\sqrt{\frac{1}{2 \pi i b}} \cdot e^{\frac{i}{2}\left(\frac{d}{b}\right) s^{s^{2}}} \int_{-\infty}^{\infty} e^{\frac{i}{2}\left(\frac{a}{b}\right) t^{2}}\left(\cos \left(\frac{s}{b} t\right)+\sin \left(\frac{s}{b} t\right)\right) f(k t) d t
$$

Putting $k t=T \Rightarrow t=\frac{T}{k} \Rightarrow d t=\frac{1}{k} d T$

$$
\begin{aligned}
& =\sqrt{\frac{1}{2 \pi i b}} \cdot e^{\frac{i}{2}\left(\frac{d}{b}\right) s^{2}} \int_{-\infty}^{\infty} e^{\frac{i}{2}\left(\frac{a}{b}\right) \frac{T^{2}}{k^{2}}}\left(\cos \left(\frac{s}{b} \frac{T}{k}\right)+\sin \left(\frac{s}{b} \frac{T}{k}\right)\right) f(T) \frac{d T}{k} \\
& =\sqrt{\frac{1}{2 \pi i b}} \cdot e^{\frac{i}{2}\left(\frac{d}{b}\right) s^{2}} \int_{-\infty}^{\infty} e^{\frac{i}{2}\left(\frac{a}{b k^{2}}\right) T^{2}}\left(\cos \left(\frac{s}{b k} T\right)+\sin \left(\frac{s}{b k} T\right)\right) f(T) \frac{d T}{k} \\
& =\frac{1}{k} \sqrt{\frac{1}{2 \pi i b}} \cdot e^{\frac{i}{2}\left(\frac{d}{b}\right) s^{2}} \int_{-\infty}^{\infty} e^{\frac{i}{(2}\left(\frac{a}{b k}\right) T^{2}}\left(\cos \left(\frac{s}{b k} T\right)+\sin \left(\frac{s}{b k} T\right)\right) f(T) \cdot e^{-\frac{i}{2}\left(\frac{a}{b k}\right) T^{2}} \cdot e^{\frac{i}{2}\left(\frac{a}{b}\right){\frac{T}{k^{2}}}^{2}} d T \\
& =\frac{1}{k} \sqrt{\frac{1}{2 \pi i b}} \cdot e^{\frac{i}{2}\left(\frac{d}{b k}\right) s^{2}} \cdot e^{-\frac{i}{2}\left(\frac{a}{b k}\right) s^{2}} \cdot e^{\frac{i}{2}\left(\frac{d}{b}\right) s^{2}} \int_{-\infty}^{\infty} e^{\frac{i}{2}\left(\frac{a}{b k}\right) t^{2}}\left(\cos \left(\frac{s}{b k} t\right)+\sin \left(\frac{s}{b k} t\right)\right) f(t) \cdot e^{-\frac{i}{2}\left(\frac{a}{b k}\right) t^{2}} \cdot e^{\frac{i}{2}\left(\frac{a}{b}\right) \frac{t^{2}}{k^{2}}} \cdot d t \\
& =\frac{1}{k} \sqrt{\frac{1}{2 \pi i b}} \cdot e^{\frac{i}{2}\left(\frac{d}{b k}\right) s^{2}} \cdot e^{\left(1-\frac{1}{k}\right) \frac{i}{2}\left(\frac{d}{b}\right) s^{2}} \cdot \int_{-\infty}^{\infty} e^{\frac{i}{2}\left(\frac{a}{b k}\right) t^{2}}\left(\cos \left(\frac{s}{b k} t\right)+\sin \left(\frac{s}{b k} t\right)\right)\left[f(t) \cdot e^{\left(\frac{1}{k}-1\right) \frac{i}{2}\left(\frac{a}{b k}\right) t^{2}}\right] d t \\
& \{C H T[f(k t)]\}(s)=\frac{1}{k} e^{\left(1-\frac{1}{k}\right) \frac{i}{2}\left(\frac{d}{b}\right) s^{2}}\left[C H T\left\{f(t) \cdot e^{\left(\frac{1}{k}-1\right) \frac{i}{2} \frac{a}{b k} t^{2}}\right\}\right]\left(\frac{s}{b k}\right)
\end{aligned}
$$

III. Conclusion:

In this paper, brief introduction of the generalized canonical Hartley transform are given and its Inversion theorem, Differentiation, Linearity, Shifting, Scaling property for canonical Hartley transform obtained which will be useful in solving differential equations occurring in signal processing and many other branches of engineering. 


\section{References:}

[1] Almeida, L.B., (1994): The fractional Fourier Transform and time- frequency representations, IEEE. Trans. on Sign. Proc., Vol. 42, No.11, 3084-3091.

[2] Bhosale B.N. and Choudhary M.S. (2002): Fractional Fourier transform of distributions of compact support, Bull. Cal. Math. Soc., Vol. 94, No.5, 349-358.

[3] Gudadhe A. S., Joshi A.V. "Generalized Canonical Cosine Transform” (August - 2012), International Journal of Engineering Research \& Technology (IJERT) Vol. 1 Issue 6.

[4] Gudadhe A. S., Joshi A.V. "Operation Transform Formulae for the Generalized Half Canonical Sine Transform" (2013), Applied Mathematical Sciences, Vol. 7, no. 21, 1033 - 1041 HIKARI Ltd

[5] Jimenez C., Torres C,, Mattos L: Fractional Hartley transform applied to optical image encryption, Journal of Physics: Conference Series 274 (2011)

[6] Moshinsky, M.(1971): Linear canonical transform and their unitary representation, Jour. Math, Phy., Vol.12, No. 8, P. $1772-1783$.

[7] Namias V. (1980): The fractional order Fourier transform and its applications to quantum mechanics, Jour. Inst. Math's. App., Vol. 25, 241-265.

[8] Pei and Ding, (2002): Eigenfunctions of Linear Canonical Transform Vol. 50, No.1.

[9] Pie and Ding, (2002): Fractional cosine, sine and Hartley Transforms, IEEE. Trans. On Sign. Proc. Vol. 50, No.7, 1661-1680.

[10] Sontakke, Gudadhe (2009): Convolution and Rayleigh's Theorem For Generalized Fractional Hartley Transform, EJPAM Vol. 2, No. $1,(162-170)$ 